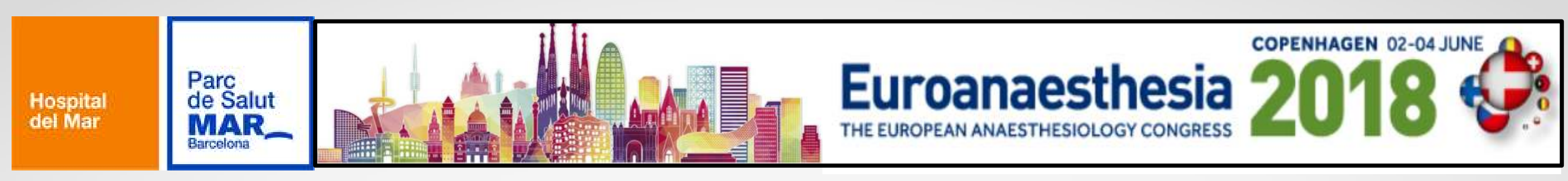

\title{
Clinical relevance of postoperative second dose of tranexamic acid in total knee arthroplasty surgery. Preliminary results of a clinical assay
}

A. Pardo Bedia, C. Luis, C. Teixell, C. Moreno, F. J. Santiveri, E. Bisbe - Department of Anaesthesia and Intensive Care Medicine. Parc de Salut Mar, Universitat Autònoma de Barcelona, Spain.

\section{Background and Goal of Study}

Tranexamic acid (TXA) has already demonstrated its efficacy and safety in reducing blood loss and transfusion in total knee arthroplasty (TKA $)^{1}$. The aim of this study is to compare the efficacy and clinical relevance of adding a second dose of TXA ${ }^{2}$ three hours after TKA within a patient blood management program (PBMP), and its impact on outcomes.

\section{Materials and Methods}

A prospective, double-blind, placebo-controlled clinical trial (EudraCT:2016- 000071-24) was conducted on the first 99 adult patients scheduled for unilateral TKA with tourniquet, between April 2016 to November 2017. Patients were randomized into two groups: Group 1 received an only intraoperative dose of TXA (15 mg/Kg) while Group 2 received an additional second dose of TXA $(10 \mathrm{mg} / \mathrm{Kg}) 3$ hours after surgery. Complications were collected within up to 30 days post-surgery.

\section{Results and Discussion}

Both groups were comparable according to gender, age, weight, surgery duration, ASA, preoperative hemoglobin $(\mathrm{Hb})$ and type of anaesthesia.

\begin{tabular}{|l|c|c|c|}
\hline & $\begin{array}{c}\text { Group 1 } \\
(\mathbf{n}=51)\end{array}$ & $\begin{array}{c}\text { Group 2 } \\
(\mathbf{n}=\mathbf{4 8})\end{array}$ & $\mathbf{p}$ \\
\hline $\mathbf{2 4 h ~ H b}(\mathbf{g} / \mathbf{d L})$ & $11.60 \pm 1.26$ & $12.01 \pm 1.20$ & 0.09 \\
\hline $\begin{array}{l}\text { Visible blood } \\
\text { loss (ml) }\end{array}$ & $185.37 \pm 132.01$ & $168.96 \pm 128.94$ & 0.53 \\
\hline $\begin{array}{l}\text { Hb discharge } \\
(\mathbf{g} / \mathbf{d L})\end{array}$ & $\mathbf{1 0 . 3 2 \pm 1 . 2 0}$ & $\mathbf{1 0 . 9 2 \pm 1 . 0 6}$ & $\mathbf{0 . 0 1}$ \\
\hline $\begin{array}{l}\text { Calculated } \\
\text { blood loss (ml) }\end{array}$ & $1674.88 \pm 604.80$ & $1482.73 \pm 520.70$ & 0.09 \\
\hline Transfusion (\%) & 1 & 0 & 0.33 \\
\hline
\end{tabular}

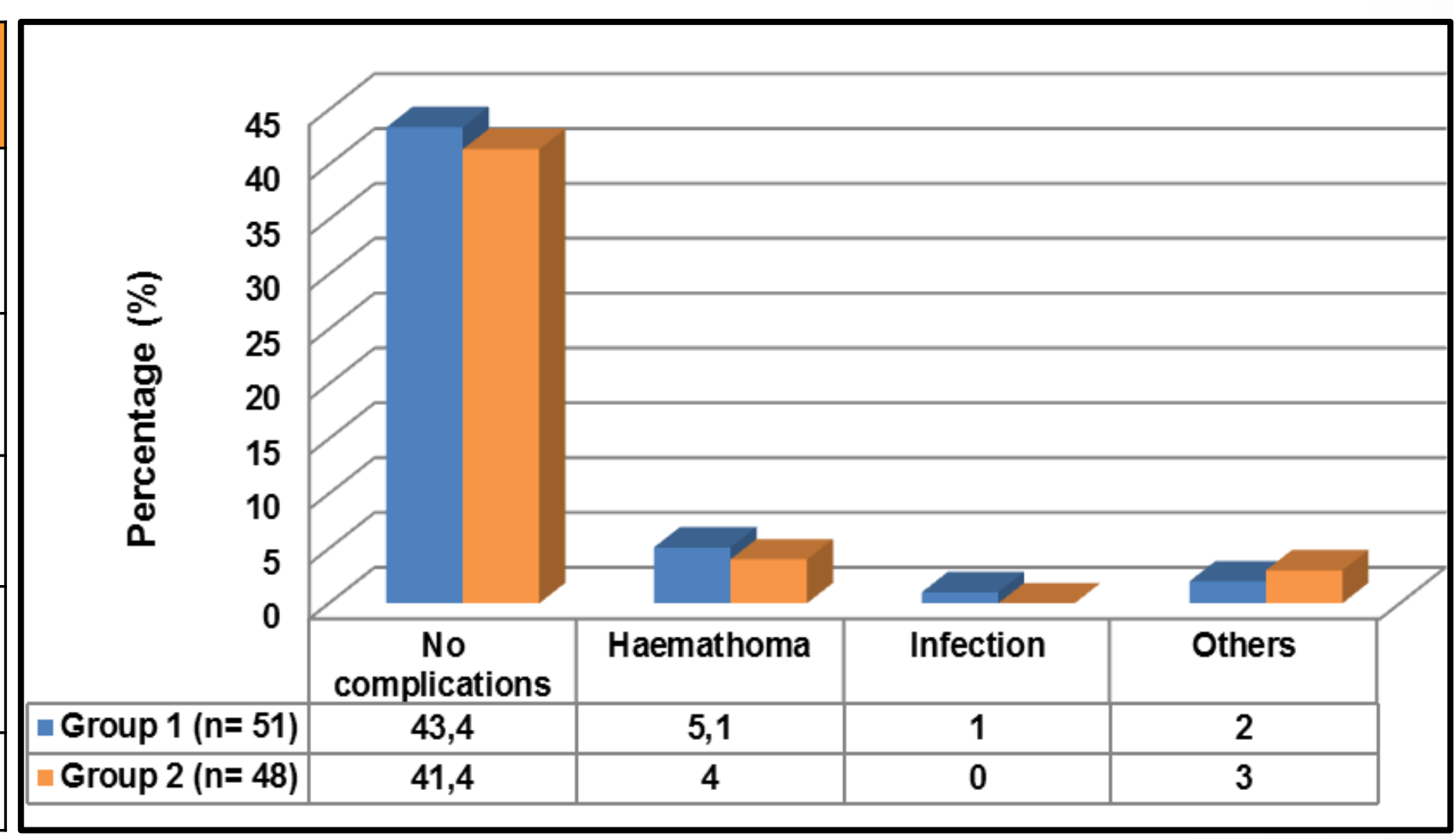

Table. Results after comparing the analysed variables between both groups.

Graphic. Complications within 30 days of surgery in both groups.

An association was found between the administration of a second dose of TXA three hours after surgery (Group 2) and a decrease in visible blood loss, calculated blood loss, and transfusion. Both 24h after surgery and at discharge hemoglobine were higher in Group 2, although $\mathrm{Hb}$ at discharge was the only variable to be statistically significant $(p=0.01)$. The second dose of TXA was not related with more complications than in Group 1.

\section{Conclusion}

Despite the fact that these are preliminary results of a larger clinical trial, a second dose of TXA after surgery showed a reduction in blood loss and a higher hemoglobin at discharge. The clinical relevance was low as there was not impact neither on transfusion nor complications. However, frail elderly patients with low hemoglobin could benefit of a second dose of TXA in order to have a greater hemoglobin at discharge.

\section{References}

1. Pitta, M., Zawadsky, M. et al. Intravenous administration of tranexamic acid effectively reduces blood loss in primary total knee arthroplasty in a 610-patient consecutive case series. Transfusion. 2016; 56: 466-471.

2. Iwai T, Tsuji S, et al. Repeat-dose intravenous tranexamic acid further decreases blood loss in total knee arthroplasty. International Orthopaedics. 2013;37(3):441-445. 